\title{
EL EJERCICIO DEL LIDERAZGO Y SU RELACIÓN CON LA INTELIGENCIA EMOCIONAL EN LA ADMINISTRACIÓN EDUCATIVA
}

M.Sc. Ileana Vargas Jiménez ${ }^{1}$

\section{Resumen}

Este artículo plantea elementos claves acerca de lo que concieme al liderazgo y su definición. Se retoman algunas investigaciones que se han realizado y cómo impacta en el manejo de las instituciones. Posteriormente, se señalan algunos planteamientos en relación con la inteligencia emocional para tratar de relacionar estos conceptos con la gestión educativa.

\section{Abstract}

This article addresses the definition of some of the key elements of leadership. It also deals with research on the issue and its impact in the management of institutions. Additionally there is a discussion concerning the emotional intelligence, relating these concepts to the tasks of education.

Palabras claves: inteligencia emocional, liderazgo, administración escolar.

\section{Importancia del ejercicio del liderazgo en la conducción escolar}

L as escuelas, como todas las organizaciones, están experimentando cambios radicales con la manera de llevar a cabo sus asuntos; es decir, requieren de directores educativos que le impregnen a las instituciones ideas innovadoras y se podría afirmar que eso está vinculado con lo que se ha venido desarrollando en los últimos tiempos, y que ciertamente deben ser

\footnotetext{
'La autora es académica de la División de Educación para el Trabajo en donde actualmente se desempeña como directora.

${ }^{2}$ Se hará referencia a este término a lo largo del artículo.
} 
consideradas como el punto de partida para el crecimiento institucional, tanto en el aspecto pedagógico como en el campo de la gerencia. El liderazgo y la inteligencia emocional son dos componentes esenciales para lograr una adecuada conducción de las escuelas.

El liderazgo, más que la gestión, es la llave del éxito organizacional, según diferentes autores; por ejemplo, Adair citado por Whitaker (1998) plantea primero que las organizaciones se componen principalmente del recurso humano y que este debe ser considerado como factor principal en la toma de decisiones administrativas. Como segundo elemento, destaca que el liderazgo es una función de cada participante de la organización, esto quiere decir, que todos tenemos capacidad de liderazgo y podemos ejercerlo en diferentes aspectos de la vida. El liderazgo es dinámico y está orientado hacia el futuro, preocupado por el progreso, el desarrollo y la excelencia.

Existe la tendencia a pensar, sin embargo, que el liderazgo sólo puede considerarse como una función que tienen aquellos que se desempeñan en determinadas instituciones y organizaciones; esto hace suponer que el liderazgo es algo que se puede ejercer si se ocupa una posición de poder y autoridad. La autoridad es cuando se asume un cargo y la persona ejerce automáticamente el ejercicio del poder, es decir, tiene la autoridad que le confiere el puesto.

\section{¿Cómo se relaciona el ejercer la dirección escolar con el liderazgo?}

Desde el punto de vista de la investigadora, se establecerá un análisis descriptivo acerca de las funciones que se manejan por un lado en la dirección sin liderazgo y cómo se comportaría un administrador con características de líder, el cual se resume en la tabla 1.

Estas son diferencias que no pretenden asegurar que el liderazgo es más importante qũe la dirección; por el contrario, la idea es buscar un entendimiento entre el aspecto humano con los procesos interactivos en las organizaciones. La actividad de la dirección es necesaria para mantener, de forma eficaz, el funcionamiento de la organización, de modo que los planes lleguen a realizarse, los procedimientos se den de la mejor manera posible, para asegurar el cumplimiento de los objetivos y que estos se logren a través de un auténtico liderazgo.

Por otro lado, existe el liderazgo transformacional, se ocupa de crear ciertas condiciones en las que todos los miembros de la organización puedan dar lo mejor de sí mismos en un clima de compromiso y desafío; en otras palabras, la dirección permite que una organización funcione, y si hay liderazgo, se ayuda a que funcione bien. 


\section{Tabla 1}

\begin{tabular}{|l|l|}
\hline \multicolumn{1}{|c|}{ Dirección sin liderazgo } & \multicolumn{1}{|c|}{ Dirección con liderazgo: } \\
\hline $\begin{array}{l}\text { Se interesa por estructuras rígidas ya es- } \\
\text { tablecidas. }\end{array}$ & $\begin{array}{l}\text { Se interesa por el comportamiento per- } \\
\text { sonal e interpersonal. }\end{array}$ \\
Mantener las funciones diarias. & $\begin{array}{l}\text { Se construye la misión y visión con todo } \\
\text { el personal. }\end{array}$ \\
Asegurar que el trabajo se haga. & $\begin{array}{l}\text { Se motiva a las personas hacia el logro } \\
\text { y se estimulan. }\end{array}$ \\
Controlar resultados y consecuencias. & $\begin{array}{l}\text { Trabajo en equipo los resultados se } \\
\text { comparten entre todos. }\end{array}$ \\
\hline
\end{tabular}

En la tabla 2, se explican las acciones que genera un líder burocrático que sería el que se desempeña en la actualidad y. por otro lado, las que genera el líder transformacional que, para el caso que nos ocupa, es el ideal en toda organización educativa.

Como se puede apreciar se establece que en las instituciones escolares se necesita de un director con características de liderazgo transformacional, ya que genera confianza, estima y, sobre todo. aprecio a los docentes.

\section{¿Qué es el Liderazgo?}

Es un término que encierra mucha polémica, porque existen diversas investigaciones que señalan diferentes aspectos: es una palabra compuesta por muchos matices entre los cuales se encuentran: la capacidad de construir un liderazgo compartido fundamentado en la participación, es decir, crea condiciones para que los colaboradores definan con él la misión y visión de la organización, así como, el consenso sobre los valores que deben dar sentido a la institución.

Adair, citado por Whitaker (1998 p.102). identifica algunas características distintivas en el concepto de liderazgo: dirección, los líderes se dedican a encontrar modos de generar una sensación clara de movimiento y dirección, lo que genera la creación de nuevas metas. nuevos servicios y estructuras 


\begin{tabular}{|l|l|}
\hline \multicolumn{1}{|c|}{ Líder burocrático } & \multicolumn{1}{|c|}{ Líder transformacional } \\
\hline $\begin{array}{l}\text { Le preocupan los papeles, circulares, la } \\
\text { rutina de la función que ejerce. }\end{array}$ & $\begin{array}{l}\text { Le interesan las personas, las escucha } \\
\text { con paciencia y atención. }\end{array}$ \\
$\begin{array}{l}\text { Mantiene el statu quo. } \\
\begin{array}{l}\text { Los profesores son funcionarios, sa- } \\
\text { ben cuál es su rol y están ahí para hacia el futuro, es visionario e } \\
\text { cumplirlo. }\end{array}\end{array}$ & $\begin{array}{l}\text { involucra a los demás en su visión y mi- } \\
\text { sión. }\end{array}$ \\
$\begin{array}{l}\text { Crea cultura de la norma, la rutina es lo } \\
\text { básico. }\end{array}$ & $\begin{array}{l}\text { tan sugerencias y recomendaciones. } \\
\text { Crea cultura de la creatividad, todos } \\
\text { aportan ideas, sugerencias. }\end{array}$ \\
$\begin{array}{l}\text { Se centra en el desarrollo de estruc- } \\
\text { turas. }\end{array}$ & Se centra en el crecimiento personal. \\
\hline
\end{tabular}

Fuente: Vargas, 2003, p. 21.

dentro de las instituciones. Por otro lado, está la inspiración, que permite a los líderes tener ideas y articular pensamientos claros, fuertes y motivadores, para el equipo de trabajo, ya que crean una energía direccional. Por último, un líder permite la creación de equipos, pues ve de forma natural y efectivos para el desarrollo de la organización y además dedican su tiempo a alentarlos.

Es importante mencionar que el trabajo en equipo es considerado como una estrategia importante que produce la energía necesaria para conseguir mejores resultados, así el líder posee un prestigio ganado por su buena labor lo que le confiere respeto y autoridad; también le permite, entusiasmar y transmitir confianza, en este caso a sus compañeros profesores. Dedica tiempo y recursos a la formación continua de sus colaboradores como medio fundamental de crecimiento personal; en otras palabras, es un liderazgo con características transformacionales de las cuales se hace referencia en la tabla anterior.

Fischam (2002) dice acerca del liderazgo, que el liderazgo personal se logra cuando el individuo emprende el camino trabajando su autoestima, creatividad, visión, equilibrio y capacidad de aprender, el liderazgo interpersonal se logra posteriormente, cuando la persona domina la comunicación, aprende a dirigir a otros a trabajar en equipo y a servir a sus seguidores (p. 19). 
Es decir, primero se deben trabajar aspectos que son meramente personales y que tienen que ver con una adecuada inteligencia emocional.

Dilts (1998) ha planteado otro estudio acerca del liderazgo, visto desde el punto de vista de la relación con motivar e influir a otros. Pero la pregunta que surge es ¿cuáles habilidades en los directores escolares requieren para lograr esas estrategias de liderazgo y de inteligencia emocional?

Estas habilidades son la comunicación, la interacción, la empatía, una adecuada autoestima, la confianza, las habilidades para trabajar en equipo, entre otras.

El mismo autor sostiene que se deben desarrollar en el individuo lo que denomina habilidades de liderazgo, en el sentido de que constituye uno de los conjuntos de capacidades más importantes que un mundo de cambio necesita. Al tratar de tomar las riendas de nuestro destino e intentar conducir el de nuestras familias, las comunidades, las organizaciones e instituciones educativas se hace cada vez más necesario la implementación de un liderazgo eficaz como una de las claves para nuestro éxito y la supervivencia en el futuro.

Con las definiciones anteriores, se rescata que el liderazgo se debe ver primero desde uno mismo, luego las relaciones con los demás o con los colaboradores, y los objetivos por alcanzar por los miembros de la organización. Seguidamente, se darán más detalles que explican cada una de esas habilidades.

Habilidades del yo: se refieren al modo en que se comporta el líder en determinada situación; lo facultan para la atención y la actitud hacia la tarea.

Habilidades de relación: se refiere a la capacidad para comprender, motivar -y comunicarse con los demás, tiene como resultado la capacidad de introducirse en el mundo de la otra persona, establecer sintonía entre él y su interlocutor para orientarla y que de esta forma reconozca problemas y objetivos, dado que los líderes deben alcanzar sus visiones y realizar sus misiones mediante su influencia sobre otras personas.

Habilidades de pensamiento estratégico: necesarias para definir y alcanzar objetivos y metas específicas; el pensamiento estratégico implica la capacidad de identificar un estado deseado pertinente y evaluar el estado de partida (Dilts, 1998 p. 34).

Como se puede observar, evidentemente existe una relación estrecha entre el liderazgo y la inteligencia emocional. En ese sentido, vale la pena mencionar que el liderazgo ha sido estudiado desde diversos puntos de vista durante muchos años, sin duda que un buen líder mantiene una adecuada inteligencia emocional. La pregunta que surge es ¿Qué es la inteligencia emocional? 
Los líderes en todo el mundo se están dando cuenta de que en algunos casos el prestar atención a los sentimientos ayuda a ahorrar tiempo, aprovechar más oportunidades y concentrar las energías en la consecución de mejores resultados. Los investigadores sugieren que muchas de las mejores decisiones de las organizaciones más dinámicas y de éxito se deben, en parte. a la inteligencia emocional.

Se define la inteligencia emocional como la habilidad de percibir, entender y aplicar efectivamente el poder de las emociones para inspirar confianza, iniciativa, energía, creatividad y motivación en los demás; para Goleman (1999), es la capacidad para reconocer sentimientos en sí mismo y en otros, siendo hábil para reconocerlos al trabajar con otros.

\section{¿Cómo se interrelaciona el liderazgo con la inteligencia emocional?}

Al ser el liderazgo una actividad basada en la relación con otros y al requerir de un control emocional inteligente, resulta claro que hay varios aspectos de la inteligencia emocional que pueden ayudar al líder a cumplir mejor sus objetivos y obligaciones. Al respecto, Reig (2004) analiza que los líderes que desean que sus seguidores aprendan a aprender, necesitan ofrecerles confianza, autocontrol, relación, capacidad de comunicación y cooperación.

El adecuado manejo de la comunicación es indispensable para todo administrador especialmente en la educación, ya que se deben generar espacios para la interacción, así como crear las condiciones necesarias para la buena marcha de la institución escolar, lo cual se logra a través de comunicación. La empatía se entiende como la posibilidad de que la persona que se está comunicando con su interlocutor siente lo que le pasa a la otra persona; esto significa, aquellos que trabajan en el campo de la administración educativa. han de estar atentos, por ejemplo, a las pistas emocionales cuando se comunican con los demás, también, tratar de mantener la calma y de serenarse en momentos difíciles; no obstante, lo anterior requiere de una gran disciplina.

La automotivación significa que todo lo que nos propongamos está regido por la motivación; es decir, desde tomar una decisión hasta ejecutarla requiere de la motivación; es el motor que nos impulsa, el estado de ánimo en que nos encontramos diariamente.

El manejo de las relaciones interpersonales es conocer las propias emociones, la conciencia de uno mismo, el poder reconocer un sentimiento mientras ocurre. Por último, el manejo de las relaciones intrapersonales se basa en la capacidad anterior, es decir, mantener un buen equilibrio emocional. 
Goleman (1999) comenta que un control adecuado de las relaciones interpersonales se sustenta en dos aspectos: el autocontrol y la empatía. Agrega que el niño que aprende a aprender requiere tener confianza, curiosidad, intencionalidad, autocontrol, relación, capacidad de comunicación y cooperación; de igual manera, el líder que quiere que sus colaboradores aprendan a aprender necesita ofrecer a sus seguidores confianza, autocontrol, relación, capacidad de comunicación y cooperación.

En relación con esta temática, se señalarán algunas pautas que brinda un mayor bienestar en la persona, porque alcanza niveles de satisfacción y efectividad en los grupos de acción. Según Herrera (2004 p. 5), las 7 SS de la persona competitiva y feliz son las siguientes:

1. Saludable: cuida su salud. se ejercita y alimenta adecuadamente para contar con la energía requerida en su trabajo intelectual, emocional y físico.

2. Sereno: firme cuando ha de serlo, pero emplea autocontrol, paciencia y tacto en su actuar.

3. Sincero: actúa en sus conversaciones y acciones basado en la ética, honestidad y justicia, es abierto para expresar sus puntos de vista.

4. Sencillo: maneja sus relaciones personales y profesionales con humildad y simplicidad, reconoce que puede aprender de todo ser humano y que sus éxitos se los debe a otras personas.

5. Servicial: emplea el poder de la retribución y del servicio para llegar dentro de las necesidades de otros, sabe que a través del servicio logra una elevación espiritual que le beneficia en otros ámbitos de su vida.

6. Simpático: cortés, amable. educado en su hablar, evita la humillación, la discriminación y los juicios a priori.

7. Sinérgico: coopera y crea climas de cooperación y de ayuda mutua en sus equipos de trabajo, tanto en la familia. la escuela u otro, se maneja a sí misrno como una parte clave de un equipo y no como una pieza indispensable, esto lo hace tomar conciencia de la importancia de la coordinación, el apoyo, la humildad para aprender, la visión común, la creatividad y la libertad para generar sugerencias en las acciones que toman diferentes equipos humanos a los que pertenece. 


\section{Otros aspectos importantes del liderazgo y cómo aplicarlo con inteligen- cia emocional}

La inteligencia emocional se compone de cinco aspectos, los cuales se vinculan luego con el liderazgo:

Relaciones interpersonales: el líder es capaz de manejar relaciones tensas y personas difíciles. En otras palabras, maneja adecuadamente la inteligencia interpersonal.

Relaciones intrapersonales: el líder es capaz de conocer sus propias emociones y así las exterioriza en el momento y lugar apropiados. Sabe controlarse en los momentos difíciles.

Empatía: es capåz de sentir lo que le sucede a su interlocutor, especialmente cuando se dan momentos difíciles, sabe escuchar y ser comprendido por los que se encuentran a su alrededor.

El líder debe ser capaz de motivar y de automotivarse, de esta manera se asegura de que los miembros de la organización se encuentran en condiciones de trabajar en armonía y da lo mejor de sí. En consecuencia, los directores escolares se asegurarían de proporcionar el ambiente adecuado, así como las estrategias necesarias para lograr un trabajo que garantice la participación colectiva de los integrantes de la escuela.

\section{A manera de conclusión}

De acuerdo con la temática planteada, se podría determinar que un buen liderazgo significa una adecuada inteligencia emocional o una adecuada inteligencia emocional significa poseer habilidades que se requieren para mantener y prolongar el liderazgo. El manejo de las relaciones humanas, la comunicación, la empatía, el manejo adecuado de resolución de conflictos entre otros, son aspectos que cada día cobran importancia en el desarrollo de las organizaciones. Los directores escolares deben conocer y procurar alcanzar el máximo provecho de sus potencialidades, aplicando para ello la inteligencia emocional con liderazgo.

Por lo tanto, la confianza, la lealtad, la dedicación impulsan la productividad e innovación, así como los logros personales de equipos y organizaciones pueden atribuirse a los sentimientos correctamente controlados.

Se presentaron varios autores quienes han estudiado el liderazgo y sus implicaciones en el campo de las relaciones humanas e igualmente el uso 
adecuado de las emociones; se constata que, en efecto, existe relación entre ambas que logran un mejor control emocional y permite de esta forma crecer personalmente en condiciones que proporcionan a los individuos una mejor capacidad de respuesta en el manejo de las instituciones escolares. No cabe duda que cuando se abordó el asunto de las emociones, se generó un nuevo paradigma de eficacia personal lo que se constituye en un nuevo reto de interés personal. Es valioso conocer estos planteamientos, porque generan a todas luces cambios trascendentales en las instituciones en general. El liderazgo debe ser ejercido por los directores escolares, ya que es el ingrediente principal de la gestión en la creación de progreso y en la manera en que las organizaciones se desarrollan. Se propone el liderazgo transformacional como modelo en la gestión escolar, ya que se centra en la persona y sobresale el apoyo individual, el propósito de la escuela, estímulo intelectual, así como promueve una cultura de colaboración y de participación.

Sin embargo, nada de esto se puede construir si los directores no asumen su rol con liderazgo e inteligencia emocional. 


\section{REFERENCIAS}

Coleman, D. (1999). La Inteligencia Emocional. Buenos Aires: Editorial Javier Vergara.

Dilts, R. (1998). Liderazgo creativo, programación neurolingüística. (Segunda Edición). Barcelona: Urano.

Fischam, D. (2002). El camino del líder. México D. F.: Editorial Aguilar.

Herrera, J. (2004). “Inteligencia emocional”. Jordanherrera79@ hotmail.com. Valencia Venezuela.

Reigh, E. (2004). Liderazgo Emocionalmente Inteligente. México: Mc Graw Hill.

Vargas, I. (2003). "Teorías Esenciales del liderazgo". En: Revista Umbral, I Semestre, $\mathrm{N}^{\circ} \mathrm{XV}$, mayo de 2003. 19-23.

Vargas, I. (2005). "Liderazgo emocionalmente inteligente, rol del administrador educativo". Primer simposio virtual hacia la administración de la educación que necesita Costa Rica en el siglo XXI. UNED.

Whitaker, P. (1998). Cómo gestionar el cambio en contextos educativos. España: Editorial Narcea. 